\title{
Correction to: Adapting Furniture to the Child - Ergonomics as a Main Tool in a Design Project
}

Cristina Salvador

\section{Correction to:}

Chapter "Adapting Furniture to the Child - Ergonomics as a Main Tool in a Design Project" in: S. Bagnara et al. (Eds.): Proceedings of the 20th Congress of the International Ergonomics Association (IEA 2018), AISC 824, https://doi.org/10.1007/978-3-319-96071-5_131

In the original version of this book, the author-provided acknowledgments section has been added in chapter "Adapting Furniture to the Child - Ergonomics as a Main Tool in a Design Project", which is a belated correction. The book have been updated with the change. 